\title{
Secondary Prevention of Colorectal Cancer: Is There an Optimal Follow-up for Patients with Colorectal Cancer?
}

\author{
John M. Carethers
}

Published online: 13 January 2010

(C) The Author(s) 2010. This article is published with open access at Springerlink.com

\begin{abstract}
Secondary prevention of colorectal cancer, as opposed to primary prevention, indicates that a person has already had the disease and there are steps being taken to prevent cancer recurrence, usually as metachronous tumors. This generally involves annual surveillance with colonoscopy after surgical removal of the initial cancer if some aspect of the colon remains. However, some familial cases may involve other modalities, such as cyclooxygenase inhibitors, as an adjunct after the initial operation. Genetic testing in suspected familial cases may identify candidates for secondary prevention. The timing for secondary prevention is critical to prevent recurrent advanced disease, which is detrimental to patient survival. Recommendations are often empiric, but some cases are based on the biological behavior of the tumor. Close follow-up with a competent health care provider, such as a gastroenterologist, is necessary to help prevent recurrence.
\end{abstract}

Keywords Colorectal cancer - Lynch syndrome .

HNPCC (hereditary nonpolyposis colorectal cancer) .

Familial cancer - Secondary prevention . Colonoscopy .

Colon cancer genetics $\cdot$ Colorectal cancer screening

\section{Introduction}

Colorectal cancer may be a devastating disease for patients if it is not discovered early in its progression. With 146,970 new cases of colorectal cancer and 49,920 deaths attributed to colorectal cancer in 2009 in the United States, prevention

\section{J. M. Carethers $(\bowtie)$}

Department of Internal Medicine, University of Michigan,

3101 Taubman Center, 1500 East Medical Center Drive,

Ann Arbor, MI 48109, USA

e-mail: jcarethe@umich.edu of this relatively common cancer will be key to reducing its incidence [1]. Indeed, some experts attribute the modest decline in colorectal cancer incidence in the United States to efforts put forth in screening the general healthy population over the age of 50 years, with removal of adenomatous polyps as its primary precursor lesion, and there is evidence for an increased colorectal cancer rate among the nonscreened population under the age of 50 years [2, 3]. Recommendations for screening have been updated recently and are based on tests that can detect cancers and those that can detect adenomatous polyps $[4 \bullet \bullet]$. These tests have varying rates for detecting colonic lesions; CT colonography and colonoscopy have the best detection rates, and colonoscopy has the added utility of removing and sampling lesions.

Secondary prevention (also known as surveillance) implies a patient has already had colorectal cancer and active steps are being taken to prevent cancer recurrence, usually as metachronous tumors. The tests used for primary screening no longer apply to secondary prevention, as direct visualization of any remaining colon is the only option in these high-risk patients $[4 \bullet \bullet, 5 \bullet, 6]$. These patients are "taken out" of the general population pool that has never had colorectal cancer and are specifically targeted for surveillance based on their history of colorectal cancer. Some aspects of primary prevention, such as eating a healthy, balanced diet (ie, low in fat, high in fiber, rich in omega-3 oil and vitamin D or calcium) and exercising regularly, still apply to secondary prevention [7]. However, these steps are not enough because biological behavior and genetic factors may predominate in the drive toward cancer recurrence, and medical surveillance by colonoscopy is critical to prevent recurrence and death $[4 \bullet \bullet, 5 \bullet, 6,7,8 \bullet$, 9 14]. In patients with inflammatory bowel disease such as ulcerative colitis, the risk of colorectal cancer begins to increase after 8 to 10 years and increases approximately $1 \%$ 
per year thereafter, particularly in patients with pancolitis [15]. In patients with ulcerative colitis, the only option for treating primary colorectal cancer is colectomy, so for these patients, secondary prevention is unnecessary.

The main risks underlying the development of recurrent tumors are largely genetic and are less likely to be environmental $[5 \bullet, 8 \bullet, 15]$. In a patient who has had colorectal cancer, the risk of a metachronous tumor is elevated, likely similar to levels of a person without cancer who has a first-degree relative under the age of 45 with colorectal cancer (a lifetime risk of $33 \%$ ) [8•]. Treatment of the initial tumor does not affect the occurrence of a second tumor; that is, although surgical resection and radiochemotherapy may treat the primary tumor, they do not prevent the occurrence of a secondary metachronous tumor $[5 \cdot, 16]$. Patients need a dedicated and consistent surveillance program to reduce their chance of death from metachronous colorectal cancer [2, 5•, 6]. The timing of secondary prevention is critical to prevent recurrent advanced disease, which is detrimental to patient survival. Recommendations often are empiric, but in some cases, they are based on the biological behavior of the tumor. In general, annual colonoscopy with removal and sampling of lesions is recommended.

\section{Secondary Prevention for Familial Colorectal Cancer Syndromes}

Familial colorectal cancer syndromes pose a particularly high risk for metachronous tumors because of the nature of germline mutations in critical growth pathways that accelerate polyp and cancer growth $[5 \cdot, 8 \bullet, 15,17]$. These syndromes include adenomatous syndromes (eg, familial adenomatous polyposis [FAP], MYH-associated polyposis, Lynch syndrome, and syndrome $\mathrm{X}$ ) that develop dysplastic polyps and pose an extremely high risk for colorectal cancer, and hamartomatous syndromes (eg, juvenile polyposis, Cowden disease, Peutz-Jeghers syndrome, and hyperplastic polyposis syndrome) that pose a variable risk for colorectal cancer development [15]. It is important to point out that many of these syndromes carry risks for other cancers besides colorectal cancer, and these cancers form the cornerstone of surveillance strategies outside the colon (Table 1).

The only endoscopic modality used for secondary prevention of colorectal cancer in the polyposis syndromes is colonoscopy $[2,5 \bullet]$. Criteria for surveilling patients and categorizing them as polyposis patients include 1) the presence of multiple polyps in the colon, 2) young age at onset of colorectal cancer, particularly age less than 50 years, 3) a strong family history suggesting a familial syndrome, and 4) evidence for a syndrome based on genetic testing [5, 8•]. In some cases, it is difficult to ascertain the potential future risk if the presenting phenotype is not clear. For

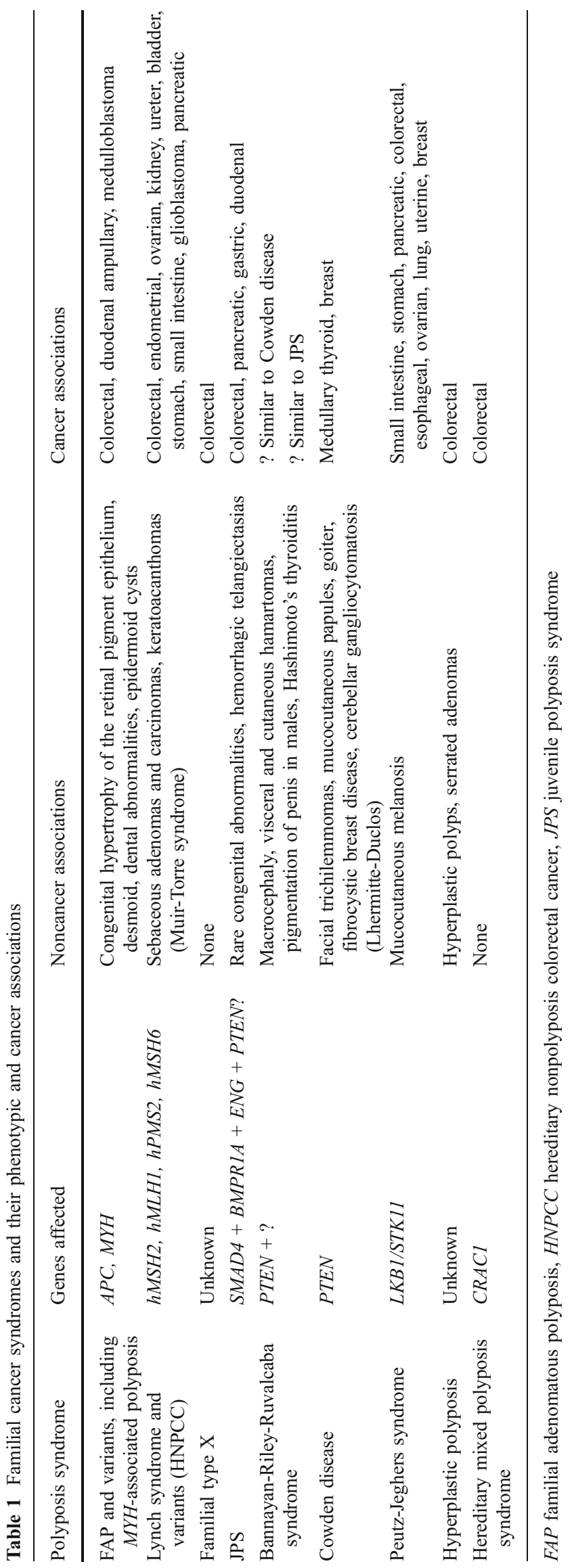


example $M Y H$-associated polyposis is transmitted in an autosomal recessive pattern so that a family history of colorectal cancer often is absent, unlike with most other polyposis syndromes [15]. Patients who present clinically with multiple colonic adenomas likely have FAP or one of its variants, and these patients often undergo total abdominal colectomy, obviating the need for secondary prevention of colorectal cancer. The phenotype of attenuated FAP or $\mathrm{MYH}-$ associated polyposis might not be recognized as such, and the initial primary tumor may be resected only regionally, as would a sporadic colorectal tumor. Furthermore, there is no precancerous phenotype for Lynch syndrome, thus other methods of recognition must be used, including family history, germline genetic testing, testing of the initial cancer for immunohistochemical expression of the DNA mismatch repair genes (Table 1), or testing for microsatellite instability $[5 \bullet, 8 \bullet$. Many Lynch syndrome patients undergo segmental resection of the colon for their initial colorectal cancer because the risk was not recognized, or in some cases in which the risk was recognized, patients may have refused a subtotal colectomy [5•]. Although colonoscopy has proven effective in increasing survival in germline-identified Lynch syndrome patients $[2,6]$, it does not result in $100 \%$ survival. Thus, many patients with adenomatous syndromes retain portions of their colon because segmental operations for their initial colorectal cancer were performed as a result of low suspicion or lack of recognition that they might have a syndrome requiring a colectomy. Likewise, segmental resections often are the norm for some hamartomatous syndromes with a variable risk for colorectal cancer, such as hyperplastic polyposis syndrome [8•, 15]. Hyperplastic polyposis syndrome may give rise to large, proximally located hyperplastic polyps that genetically follow a sessile serrated adenoma pathway [18]. In many instances of hamartomatous syndromes, secondary prevention via colonoscopy generally is adequate because of the variable nature of cancer recurrence. When the polyp burden is so high it prevents adequate and thorough sampling for the occurrence of metachronous cancer, colectomy should be strongly considered.

The timing of colonoscopy for secondary prevention in familial syndromes is largely empiric; for most syndromes, it is recommended annually (Table 2) $[4 \bullet \cdot, 5 \bullet, 15]$. However, there have been cases in which yearly examinations showed interval cancer development; these cases have occurred mostly in patients with Lynch syndrome [5•]. The nature of the genetic lesion in Lynch syndrome, loss of DNA mismatch repair function, and subsequent targeting of susceptible growth-regulating genes seem to drive cancer development rapidly, to the point at which a polyp stage, such as one would visualize with colonoscopy in a FAP patient, is rarely seen $[4 \bullet \bullet, 5 \bullet, 8 \bullet$. Furthermore, some Lynch syndrome tumors are flat, lacking an exophytic form, because of the lack of $K-R A S$ activating mutations [8• 19]. Thus, not only may cancers form rapidly in Lynch syndrome, they may be missed on colonoscopy without careful examination $[19,20 \bullet \cdot$. In the case of Lynch syndrome, careful evaluation of each patient's history, as well as careful examination of the colon, may dictate the need for colonoscopic examinations more frequently than once a year [5•]. There is at least one feature of Lynch syndrome that has led

Table 2 Suggested surveillance intervals for secondary prevention of colorectal cancer

Condition Interval

Sporadic colorectal cancer

Clearing colonoscopy within 3-6 months of initial tumor, preferably preoperatively if possible. Colonoscopy 1 year after initial tumor resection, and if exam is normal, subsequent exam may be extended to 3 years. If the 3 -year exam is normal, colonoscopy may be extended to every 5 years

FAP and variants, including $M Y H$-associated Annual colonoscopy and polypectomy of any remaining colon; if polyp burden is high, surgical removal of remainder of colon should be considered

Lynch syndrome and variants (HNPCC)

Annual colonoscopy of any remaining colon (at a minimum). Consideration should be given to resecting remainder of colon in germline-positive patients

Familial type $\mathrm{X}$

Annual colonoscopy of any remaining colon

Juvenile polyposis syndrome

Annual colonoscopy of any remaining colon; if polyp burden is high, surgical removal of remainder of colon should be considered

Bannayan-Riley-Ruvalcaba syndrome

Annual colonoscopy of any remaining colon

Cowden disease

Annual colonoscopy of any remaining colon

Peutz-Jeghers syndrome

Annual colonoscopy of any remaining colon; if polyp burden is high, surgical removal of remainder of colon should be considered

Hyperplastic polyposis

Annual colonoscopy of any remaining colon; if polyp burden is high, surgical removal of remainder of colon should be considered

Hereditary mixed polyposis syndrome

Annual colonoscopy of any remaining colon

FAP familial adenomatous polyposis, $H N P C C$ hereditary nonpolyposis colorectal cancer 
experts to suggest yearly examinations [4••]: Lynch patients with colorectal cancer, when compared stage to stage with non-Lynch patients with colorectal cancer, have better survival rates [8•]. The implication is that finding a cancer at yearly colonoscopy still may afford better survival, even though a cancer was discovered. Although the goal of secondary prevention in a familial syndrome is to find lesions at their earliest stage to prevent any deterioration in patient survival, a metachronous tumor in a patient with Lynch syndrome may still have a good outcome with appropriate treatment, which should include colectomy. Also, there is growing evidence that patients with Lynch syndrome who develop colorectal cancer, as well as those with sporadic colorectal cancer who show loss of DNA mismatch repair within their tumors, do not respond to standard 5-fluorouracil-based chemotherapy and have no increase in survival [21-25]. Thus, secondary prevention with colonoscopy followed by surgical resection if a lesion is found is the mainstay of treatment for these high-risk patients, as chemotherapy may not improve survival.

Chemoprevention has been used as an adjunct to colonoscopic secondary prevention in some FAP patients [26-28]. It should be pointed out that chemoprevention is not a substitute for colectomy; however, there is evidence that the number and size of adenoma precursors are smaller in patients receiving cyclooxygenase (COX) inhibitors [26]. On cessation of COX inhibitors, the number and size of polyps return to their pretreatment levels, indicating that the drug must be present to have an effect. COX inhibitors do not prevent the onset of precursor polyps in young FAP patients [27], and it is not clear whether they prevent metachronous tumor development in FAP patients.

\section{Secondary Prevention of Sporadic Colorectal Cancer}

Colorectal cancer patients who do not clearly fit a polyposis syndrome are said to have sporadic disease, but their risk for a metachronous colorectal cancer is elevated, likely to the level of that of a noncancer patient with a young firstdegree relative with colorectal cancer (ie, a lifetime risk of $33 \%$ ) [4••, 15]. Thus, surveillance by colonoscopy for secondary prevention of recurrent tumor is paramount, much like surveillance for primary or secondary prevention in familial patients, as outlined earlier.

There are several reasons a metachronous colorectal cancer might develop in a patient with sporadic disease: 1 ) the patient has an unrecognized familial syndrome; 2) residual tumor may be present after resection by polypectomy or at surgery; 3 ) the patient has strong risk factors that modify the local colonic environment, such as excessive serum growth hormone in acromegaly; and 4) the patient has genetic risk factors that do not fit into a familial syndrome.
To help recognize a familial syndrome, the clinician should obtain a careful family history that takes into account all types of cancers and polyps in the patient's family. Conditions such as acromegaly should be recognized, but personal genetic factors are difficult to quantify.

Residual tumor has been described with colonoscopic removal (snare polypectomy and endoscopic mucosal resection) as well as surgical removal, as a result of inadequate margins or the presence of unrecognized extensive disease at surgery. In the case of colonoscopic removal, re-colonoscopy at very short intervals, such as every few weeks to every 2 months, is recommended to reevaluate the site [4••]. It is also recommended that the site be identifiable, such as with tattooing, for the next colonoscopy to ensure precise observation of the mucosa at the resection site. Careful attention must be paid to the resected pathologic specimen, including looking for signs of vascular or lymphatic invasion, margins free of tumor, and the tumor grade [7]. If the pathology does not dictate a free-and-clear specimen, then surgical resection is recommended, followed by surveillance colonoscopy within a year. In the case of recurrence after surgical resection, colonoscopic surveillance should be combined with a radiologic examination, such as abdominal CT scanning, because of potential recurrence of tumor outside the luminal surface of the colon. At colonoscopy, the surgical anastomosis should be identified; in some cases, random biopsies of the anastomosis may be sent for pathologic examination to rule out luminal recurrence.

Recommendations for secondary prevention of colorectal cancer in patients with sporadic disease are based on the length of time from the primary tumor occurrence [4*0]. Patients - preferably before, if possible, but certainly after their initial primary colorectal cancer-should have a clearing colonoscopy to rule out synchronous tumors and polyps. Within 1 year after the primary tumor resection, another colonoscopy should take place, with careful observation of the anastomosis as well as the remainder of the mucosa for lesions that may have been missed on the previous examination. If this examination is negative for lesions in patients with sporadic disease, the next colonoscopy generally may be performed 3 years after surgical resection of the primary tumor. If that colonoscopy is negative, then secondary prevention by colonoscopy may be extended to every 5 years (Table 2$)[4 \bullet \bullet]$.

\section{Conclusions}

Secondary prevention of colorectal cancer is principally by colonoscopic visualization of any remaining colon in patients with familial or sporadic colorectal cancer. Routine screening modalities used for primary prevention have no role in secondary prevention. A key role for the clinician is 
determining whether a patient has a familial syndrome, which dictates annual surveillance by colonoscopy and subsequent colectomy if a metachronous lesion is found, or has sporadic disease, for which the patient can undergo surveillance at gradually lengthening intervals. Clues to a familial form of colorectal cancer should be actively ascertained. It is important to identify patients with Lynch syndrome, as their colorectal cancer may develop quite rapidly and their examinations for secondary prevention surveillance may have to be more frequent than once a year. Close follow-up by a competent health care provider, such as a gastroenterologist, is necessary to help prevent recurrence.

Acknowledgments This work is supported by the US Public Health Service (DK067287); the University of California, San Diego (UCSD), Digestive Diseases Research Development Center (DK080506); and the San Diego State University/UCSD Comprehensive Cancer Center Partnership (CA132379 and CA13238).

Disclosure No potential conflict of interest relevant to this article was reported.

Open Access This article is distributed under the terms of the Creative Commons Attribution Noncommercial License which permits any noncommercial use, distribution, and reproduction in any medium, provided the original author(s) and source are credited.

\section{References}

Papers of particular interest, published recently, have been highlighted as follows:

- Of importance

-. Of major importance

1. Jemal A, Siegel R, Ward E, et al.: Cancer statistics, 2009. CA Cancer J Clin 2009, 59:225-249.

2. Dove-Edwin I, Sasieni P, Adams J, Thomas HJ: Prevention of colorectal cancer by colonoscopic surveillance in individuals with a family history of colorectal cancer: 16 year, prospective, followup study. BMJ 2005, 331:1047.

3. Siegel RL, Jemal A, Ward EM: Increase in incidence of colorectal cancer among young men and women in the United States. Cancer Epidemiol Biomarkers Prev 2009, 18:1695-1698.

4. •- Levin B, Lieberman DA, McFarland B, et al.: American Cancer Society Colorectal Cancer Advisory Group; US Multi-Society Task Force; American College of Radiology Colon Cancer Committee: Screening and surveillance for the early detection of colorectal cancer and adenomatous polyps, 2008: a joint guideline from the American Cancer Society, the US Multi-Society Task Force on Colorectal Cancer, and the American College of Radiology. CA Cancer J Clin 2008, 58:130-160. This article outlines the latest screening and surveillance guidelines for colorectal cancer in the United States.

5. - Boland CR, Koi M, Chang DK, Carethers JM: The biochemical basis of microsatellite instability and abnormal immunohistochemistry and clinical behavior in Lynch syndrome: from bench to bedside. Fam Cancer 2008, 7:41-52. This article reviews major aspects of diagnosing and caring for patients with Lynch syndrome.

6. Järvinen HJ, Aarnio M, Mustonen H, et al.: Controlled 15-year trial on screening for colorectal cancer in families with hereditary nonpolyposis colorectal cancer. Gastroenterology 2000, 118:829-834.

7. Boland CR, Sinicrope FA, Brenner DE, Carethers JM: Colorectal cancer prevention and treatment. Gastroenterology 2000, 118: S115-S128.

8. - Grady WM, Carethers JM: Genomic and epigenetic instability in colorectal cancer pathogenesis. Gastroenterology 2008, 135: 1079-1099. This article is a comprehensive review of genetic changes that occur during colorectal cancer development and metastasis.

9. Carethers JM: Intersection of TGFbeta and Wnt signaling pathways in colorectal cancer and metastasis. Gastroenterology 2009, 136:33-36.

10. Chow JYC, Dong H, Quach KT, et al.: TGFb mediates PTEN suppression and cell motility through calcium-mediated $\mathrm{PKCa}$ activation in pancreatic cancer cells. Am J Physiol Gastrointest Liver Physiol 2008, 294:G899-G905.

11. Chow JYC, Cabral JA, Chang J, Carethers JM: TGF-beta modulates PTEN expression independently of SMAD signaling for growth proliferation in colon cancer cells. Cancer Biol Ther 2008, 7:1694 1699.

12. Jung BH, Beck SE, Cabral J, et al.: Activin type 2 receptor restoration in MSI-H colon cancer suppresses growth and enhances migration with activin. Gastroenterology 2007, 132:633-644.

13. Jung B, Smith EJ, Doctolero RT, et al.: Influence of target gene mutations on survival, stage and histology in sporadic microsatellite unstable colon cancers. Int J Cancer 2006, 118:25092513.

14. Carethers JM, Hawn MT, Greenson JK, et al.: Prognostic significance of allelic lost at chromosome 18q21 for stage II colorectal cancer. Gastroenterology 1998, 114:1188-1195.

15. Jung B, Boland CR, Carethers JM: Neoplasia of the gastrointestinal tract. In Textbook of Gastroenterology, edn 5. Edited by Yamada T, Alpers DH, Kaplowitz N, et al. Philadelphia: Blackwell Publishers; 2008.

16. Carethers JM: Systemic treatment of advanced colorectal cancertailoring therapy to the tumor. Ther Adv Gastroenterol 2008, $1: 33-42$.

17. Huang SC, Lavine JE, Boland PS, et al.: Germline characterization of early-aged onset of hereditary non-polyposis colorectal cancer. J Pediatr 2001, 138:629-635.

18. Makinen MJ: Colorectal serrated adenocarcinoma. Histopathology 2007, 50:31-50.

19. Yashiro M, Carethers JM, Laghi L, et al.: Genetic pathways in the evolution of morphologically distinct colorectal neoplasms. Cancer Res 2001, 60:2676-2683.

20. • Soetikno RM, Kaltenbach T, Rouse RV, et al.: Prevalence of nonpolypoid (flat and depressed) colorectal neoplasms in asymptomatic and symptomatic adults. JAMA 2008, 299:1027-1035. This article describes the importance of recognizing flat and depressed colonic lesions, as these are more likely to be cancerous.

21. Jo W-S, Carethers JM: Chemotherapeutic implications in microsatellite unstable colorectal cancer. Cancer Biomark 2006, 2:5160.

22. Carethers JM: Prospective evaluation of fluorouracil chemotherapy based on the genetic make-up of colorectal cancer [editorial]. Gut 2006, 55:759-761.

23. Carethers JM, Chauhan DP, Fink D, et al.: Mismatch repair proficiency and in vitro response to 5-fluorouracil. Gastroenterology 1999, 117:123-131.

24. Tajima A, Hess MT, Cabrera BL, et al.: The mismatch repair complex hMutSa recognizes 5-fluoruracil-modified DNA: implications 
for chemosensitivity and resistance. Gastroenterology 2004, 127: 1678-1684.

25. Carethers JM, Smith EJ, Behling CA, et al.: Use of 5-fluorouracil and survival in patients with microsatellite unstable colorectal cancer. Gastroenterology 2004, 126:394-401.

26. Steinbach G, Lynch PM, Phillips RK, et al.: The effect of celecoxib, a cyclooxygenase-2 inhibitor, in familial adenomatous polyposis. N Engl J Med 2000, 342:1946-1952.
27. Giardiello FM, Yang VW, Hylind LM, et al.: Primary chemoprevention of familial adenomatous polyposis with sulindac. $\mathrm{N}$ Engl J Med 2002, 346:1054-1059.

28. Koornstra JJ, Rijcken FE, Oldenhuis CN, et al.: Sulindac inhibits beta-catenin expression in normal-appearing colon of hereditary nonpolyposis colorectal cancer and familial adenomatous polyposis patients. Cancer Epidemiol Biomarkers Prev 2005, 14:16081612 . 